\title{
The DRBEM solution of Cauchy MHD duct flow with a slipping and variably conducting wall using the well-posed iterations
}

\author{
Cemre Aydin* [D and Munevver Tezer-Sezgin (D) \\ Department of Mathematics, Middle East Technical University, Turkey \\ e171938@metu.edu.tr,munt@metu.edu.tr
}

\section{ARTICLE INFO}

Article History:

Received 15 August 2018

Accepted 01 February 2019

Available 27 July 2019

\section{Keywords:}

DRBEM

Cauchy problem

MHD duct flow

Slip velocity

Variable conductivity

AMS Classification 2010:

65N20; 65N21; 65N38

\begin{abstract}
The present study focuses on the numerical investigation of the Cauchy Magnetohyrodynamic (MHD) duct flow in the presence of an externally applied oblique magnetic field, with a slipping and variably conducting wall portion of the duct walls. The underspecified and overspecified boundary informations for the velocity of the fluid and the induced magnetic field on both slipping and variably conducting duct wall and its opposite part, respectively, constitutes the Cauchy MHD duct flow problem. This study aims to recompute the velocity of the fluid and induced magnetic field with specified slip length and conductivity constant, respectively, on the underspecified wall which is both slipping and variably conducting. The governing coupled convection-diffusion type MHD equations for the direct and inverse formulations are solved in one stroke using the dual reciprocity boundary element method (DRBEM). Both the velocity and induced magnetic field and their normal derivatives to be used as overspecified boundary conditions for the construction of Cauchy problem are obtained through the direct formulation of the problem. The well-posed iterations are used in the regularization of the ill-conditioned systems of linear algebraic equations resulting from the DRBEM discretization of Cauchy problem (inverse problem). Numerical solutions for the slip velocity and induced magnetic field are obtained for Hartmann number values $M=5,10,50$. The main advantages of the DRBEM are its boundary only nature and the capability of providing both the unknowns and their normal derivatives on the underspecified walls so that the conductivity constant and the slip length between them can be recovered at a low computational expense.
\end{abstract}

\section{Introduction}

The effects of magnetic field through the electrically conducting fluids, such as electrolytes, blood plasmas, salt waters, liquid metals etc. are concerned by the magnetohyrodynamics (MHD). The combination of the Navier-Stokes equations of fluid dynamics with the Maxwell's equations of electromagnetism through Ohm's law describes the conducting fluid motion under the impact of externally applied magnetic field. The industrial and biological applications of the magnetohydrodynamic fluid flow in channels are generally encountered in the MHD generators, MHD pumps, accelerators, nuclear reactors, and the blood flow pressure measurements [1].

The distance from the fluid to the channel walls within the solid stage where the velocity of the flow diminished is described as the slip length. It is stated that the slip in the MHD flow will likely occur in fusion reactors with liquid metal flows in contact with ceramics as some current experimental data shows. The presence of Dirichlet/Neumann or mixed type boundary conditions

*Corresponding Author 
for the velocity $V$ and the induced magnetic field $B$ on the complete channel walls results in direct problems. The slipping velocity is defined with mixed boundary condition as $V+\alpha \frac{\partial V}{\partial n}=0$ where $\alpha$ is the slip length and the variably conducting duct wall is defined with $B+c \frac{\partial B}{\partial n}=0$ where $c$ is the conductivity constant. Exact solutions of the MHD equations exist for some particular duct geometries with no-slip and insulated or perfectly conducting duct walls [1,2]. Analytical solutions in terms of asymptotic expansions are given by Ligere et. al [3,4] for MHD duct flow with perfectly conducting Hartmann walls and slipping side walls. The MHD equations are commonly solved using numerical methods in no-slip and most general form of the wall conductivity conditions [5] 8 . In some engineering applications some parts of the boundary may allow both the velocity slip and conductivity change depending on the material it is made of. Slip length and conductivity constant on these parts of the boundary can be determined when the MHD flow problem is designed as a Cauchy problem. In this case, the boundary conditions for the velocity of the fluid can be incomplete either in the form of underspecified or overspecified on different parts of the boundary. These are called inverse problems or Cauchy problems and it is well-known that they are generally ill-posed [9]. Therefore, to solve such kind of problems, a regularization technique must be used.

The DRBEM transforms the differential equations defined in the problem region into integral equations defined on the boundary, approximating also the inhomogeneities of the equations using radial basis functions which are related to differential operator with particular solutions. By this way, a system of discretized equations for the boundary nodes and at some selected interior points is solved [10]. One main advantage of BEM or DRBEM is to provide both the unknown and its normal derivative on the boundaries. There are quite a number of BEM or DRBEM solutions of MHD duct flow problems with no-slip velocity condition and different combinations of wall conductivities [5, 6, 8, 11, 12]. In the studies carried by S. Smolentsev and E. Ligere [4,13, MHD flow problems with slip velocity conditions and with a known slip length in a channel are solved analytically on various parts of the duct walls. The DRBEM solution of Cauchy MHD duct flow equations with a perturbed slipping upper boundary is given in [14 by solving the MHD equations as a whole.
In this paper, the numerical solutions of the direct and Cauchy MHD flow problems are accomplished when one of the channel walls contains both the slip and variably conducting conditions. The slip length and the conductivity constant are assumed to be unknown in the inverse or Cauchy problem and thus, both the velocity, the induced magnetic field and their normal derivatives are going to be determined which are underspecified boundary conditions on that part of the duct walls. When the direct problem is solved for a specified slip length and conductivity constant with Dirichlet type velocity and induced magnetic field conditions, the opposite side of the duct wall contains overspecified conditions (both the velocity, induced magnetic field and their normal derivatives obtained from the direct DRBEM solution). The well-posed iterations are employed to regularize the discretized ill-posed problem resulting from the discretization of Cauchy MHD problem. The numerical results are performed for direct and inverse problems for Hartmann number values $M=5,10,50$, and the regularization method is examined in terms of convergence to the solution of direct problem obtained with an estimated slip length and conductiviy constant. Also, the slip length and the conductivity constant are regained through the Cauchy MHD flow problem solution on the slipping and variably conducting wall. The DRBEM has the advantage of discretizing only the boundary and providing both the unknowns and their normal derivatives on the boundary which form overdetermined boundary information for the Cauhcy MHD flow problem. Thus, it enables us to obtain the solution of Cauchy MHD flow problem at a small computational expense.

\section{Mathematical formulation of the problem}

An electrically conducting fluid is flowing in a long pipe of rectangular cross-section (duct) with a pressure gradient $\frac{\partial p}{\partial z}$, and an external uniform magnetic field is applied by forming an angle $\beta$ with the y-axis. The flow becomes fully developed in the direction of pipe-axis and the problem reduces to two-dimension in the duct. Thus, the two-dimensional, steady and fully developed MHD flow in a rectangular duct under the effect of an externally applied oblique magnetic field is considered [6].

The left vertical wall allows the slip of the fluid and also variable conductivity. The nondimensional governing equations for the velocity 
$V(x, y)$ of the fluid and the induced magnetic field $B(x, y)$ are [1]

$$
\begin{array}{r}
\nabla^{2} V+M_{x} \frac{\partial B}{\partial x}+M_{y} \frac{\partial B}{\partial y}=-1 \\
\quad \text { in } \quad-1<x, y<1
\end{array}
$$$$
\nabla^{2} B+M_{x} \frac{\partial V}{\partial x}+M_{y} \frac{\partial V}{\partial y}=0
$$

where $M_{x}=M \sin (\beta), M_{y}=M \cos (\beta)$ and $M=L B_{0} \sqrt{\sigma / \nu \rho}=\sqrt{\left(M_{x}\right)^{2}+\left(M_{y}\right)^{2}}$ is the Hartmann number resulted during the nondimensionalization of the equations, and $L, B_{0}$, $\sigma, \nu$ and $\rho$ are the characteristic length, the external magnetic field intensity, electrical conductivity, kinematic viscosity, and the density of the fluid, respectively. The magnetic field applies in the direction obtained with an angle $\beta$ from the $y$-axis.

The physical configuration of the MHD duct flow problem with a slipping and variably conducting left wall leads to the boundary conditions as

$$
\begin{aligned}
& V=0, B=0 \quad \text { on } \quad y=\mp 1, \quad-1<x<1 \\
& V=0, B=0 \quad \text { on } \quad x=1, \quad-1<y<1 \\
& V+\alpha \frac{\partial V}{\partial n}=0 \quad \text { on } \quad x=-1, \quad-1<y<1 \\
& B+c \frac{\partial B}{\partial n}=0 \text { on } \quad x=-1, \quad-1<y<1 .
\end{aligned}
$$

The main purpose of the study is to regain the slip length and conductivity constant on the slipping and variably conducting portion (left wall) of the duct walls. Hence, the MHD duct flow problem is constructed as a Cauchy problem in terms of the velocity $V(x, y)$ and the induced magnetic field $B(x, y)$ as direct and inverse problems configured in Figure 1,
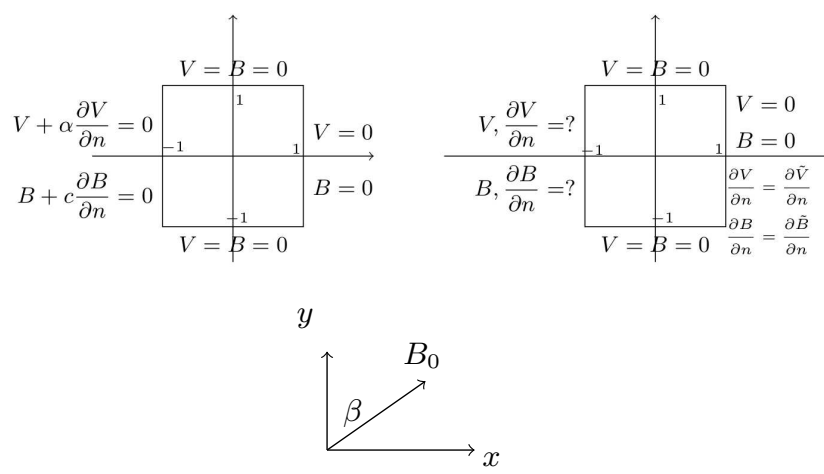

Figure 1. Boundary conditions for the direct (left) and the Cauchy (right) problems. $\tilde{V}, \tilde{B}$ denote direct problem solution. $\alpha$ is the dimensionless slip length and $c$ is the conductivity constant which are going to be gained from the inverse formulation of the problem. First the MHD problem (11)-(2) is going to be solved for specific values of $\alpha$ and $c$. Then, with the obtained normal derivative values of the velocity and the induced magnetic field on the left wall, the Cauchy problem is constructed.

\section{The DRBEM application}

The DRBEM is applied to the MHD differential equations (1) by using the fundamental solution of the Laplace equation which is $u^{*}=\ln \left(\frac{1}{r}\right) / 2 \pi,[10$. Therefore, the terms other than Laplacian are considered as inhomogeneity and by weighting the equations (1) by $u^{*}$ and applying Green's second identity two times, we obtain the following equations

$$
\begin{aligned}
& c_{i} V_{i}+\int_{\Gamma} q^{*} V d \Gamma-\int_{\Gamma} u^{*} \frac{\partial V}{\partial n} d \Gamma= \\
& \int_{\Omega}\left(-1-M \frac{\partial B}{\partial x}-M_{y} \frac{\partial B}{\partial y}\right) u^{*} d \Omega=\int_{\Omega} b_{1} u^{*} d \Omega \\
& c_{i} B_{i}+\int_{\Gamma} q^{*} B d \Gamma-\int_{\Gamma} u^{*} \frac{\partial B}{\partial n} d \Gamma= \\
& \int_{\Omega}\left(-M_{x} \frac{\partial V}{\partial x}-M_{y} \frac{\partial V}{\partial y}\right) u^{*} d \Omega=\int_{\Omega} b_{2} u^{*} d \Omega
\end{aligned}
$$

where $q^{*}=\frac{\partial u^{*}}{\partial n}, \Gamma$ is the boundary $x=\mp 1, y=$ $\mp 1$, and the index $i$ denotes the source point. The constant $c_{i}$ is $1 / 2$ and 1 when the source point is on the boundary and in the interior of the domain, respectively.

The right hand side domain integrals contain the inhomogeneities of equations (1) which can be approximated by radial basis functions, e.g, $f(r)=1+r$ which are connected to particular solutions $\hat{u}_{j}$ 's with the equation $\nabla^{2} \hat{u}_{j}=f_{j}$. The approximations of the integrands in the domain integrals are given by $\sum_{j=1}^{N+L} \alpha_{j} f_{j}$ and $\sum_{j=1}^{N+L} \beta_{j} f_{j}$ for the equations (3) and (4), respectively, where $\alpha_{j}$ 's and $\beta_{j}$ 's are undetermined coefficients. The radial basis functions are collocated at the discretized points as $f_{i j}=1+r_{i j}$ giving the coordinate matrix $F=\left(f_{i j}\right)$ where $r_{i j}$ is the distance between the nodes $\mathrm{i}$ and $\mathrm{j}, N$ and $L$ denote the number of boundary and interior nodes, respectively, when the boundary $\Gamma$ is discretized using $N$ constant boundary elements. The collocation of the inhomogeneities at $N+L$ points results in the systems $F \alpha=b_{1}, F \beta=b_{2}$.

Then, the right hand sides of the equations (3)-(4) are rewritten as 


$$
\begin{aligned}
c_{i} V_{i}+\int_{\Gamma} q^{*} V d \Gamma-\int_{\Gamma} u^{*} \frac{\partial V}{\partial n} d \Gamma= \\
\sum_{j=1}^{N+L} \alpha_{j}\left(c_{i} \hat{u}_{i j}+\int_{\Gamma} q^{*} \hat{u}_{j} d \Gamma-\int_{\Gamma} u^{*} \frac{\partial \hat{u}_{j}}{\partial n} d \Gamma\right)
\end{aligned}
$$

$$
\begin{aligned}
c_{i} B_{i}+\int_{\Gamma} q^{*} B d \Gamma-\int_{\Gamma} u^{*} \frac{\partial B}{\partial n} d \Gamma= \\
\sum_{j=1}^{N+L} \beta_{j}\left(c_{i} \hat{u}_{i j}+\int_{\Gamma} q^{*} \hat{u}_{j} d \Gamma-\int_{\Gamma} u^{*} \frac{\partial \hat{u}_{j}}{\partial n} d \Gamma\right)=
\end{aligned}
$$

by applying the BEM also to the inhomogeneities connected to the same Laplace operator as $\nabla^{2} \hat{u}_{j}=f_{j}$. The discretization of the boundary results in a system of matrix vector equations

$$
\begin{aligned}
& H V-G \frac{\partial V}{\partial n}= \\
& (H \hat{U}-G \hat{Q}) F^{-1}\left\{-1-M_{x} \frac{\partial B}{\partial x}-M_{y} \frac{\partial B}{\partial y}\right\} \\
& H B-G \frac{\partial B}{\partial n}= \\
& \quad(H \hat{U}-G \hat{Q}) F^{-1}\left\{-M_{x} \frac{\partial V}{\partial x}-M_{y} \frac{\partial V}{\partial y}\right\} .
\end{aligned}
$$

The matrices $\hat{U}, \hat{Q}$ and $F$ are constructed by taking each of the vectors $\hat{u}_{j}, \hat{q}_{j}$ and $f_{i j}=f_{j}\left(r_{i}\right)$ as columns, respectively. The components of the $H$ and $G$ matrices are given for constant elements as

$$
\begin{aligned}
H_{i j} & =c_{i} \delta_{i j}+\frac{1}{2 \pi} \int_{\Gamma_{j}} \frac{\partial}{\partial n}\left(\ln \left(\frac{1}{r}\right)\right) d \Gamma_{j} \\
H_{i i} & =-\sum_{j=1, j \neq i}^{N} H_{i j} \\
G_{i j} & =\frac{1}{2 \pi} \int_{\Gamma_{j}} \ln \left(\frac{1}{r}\right) d \Gamma_{j} \\
G_{i i} & =\frac{l}{2 \pi}\left(\ln \left(\frac{2}{l}\right)+1\right)
\end{aligned}
$$

where $l$ is the length of the elements and $\delta_{i j}$ is the Kronecker delta function.

The space derivatives for $V$ and $B$ are computed by using the coordinate matrix as

$$
\begin{aligned}
& \frac{\partial V}{\partial x}=\frac{\partial F}{\partial x} F^{-1} V, \\
& \frac{\partial B}{\partial x}=\frac{\partial F}{\partial x} F^{-1} B, \\
& \frac{\partial V}{\partial y}=\frac{\partial F}{\partial y} F^{-1} V,
\end{aligned}
$$

$$
\frac{\partial B}{\partial y}=\frac{\partial F}{\partial y} F^{-1} B
$$

where the coordinate matrix $F$ is invertible since the leading diagonal of $F$ is nonzero [10]. The coupled matrix-vector equations (77) and (8) can be solved together by constructing the whole system as

$$
\left[\begin{array}{ll}
H_{N+L \times N+L} & K_{N+L \times N+L} \\
K_{N+L \times N+L} & H_{N+L \times N+L}
\end{array}\right]\left[\begin{array}{l}
V_{N+L \times 1} \\
B_{N+L \times 1}
\end{array}\right]
$$

$$
\left[\begin{array}{ll}
G_{N+L \times N+L} & 0_{N+L \times N+L} \\
0_{N+L \times N+L} & G_{N+L \times N+L}
\end{array}\right]\left[\begin{array}{l}
\frac{\partial V}{\partial n} \\
N+L \times 1 \\
\frac{\partial B}{\partial n}{ }_{N+L \times 1}
\end{array}\right]+
$$

$$
\left[\begin{array}{l}
b_{N+L \times 1} \\
0_{N+L \times 1}
\end{array}\right]
$$

where $K=(H \hat{U}-G \hat{Q}) F^{-1}\left(M_{x} \frac{\partial F}{\partial x} F^{-1}+\right.$ $\left.M_{y} \frac{\partial F}{\partial y} F^{-1}\right)$ and $b=-(H \hat{U}-G \hat{Q}) F^{-1}$.

The solution of the above matrix-vector equation gives the unknown vectors $V, B, \frac{\partial V}{\partial n}$ and $\frac{\partial B}{\partial n}$ which are $(N+L) \times 1$ vectors on each part of the boundary.

\section{The well-posed iterations}

For the inverse MHD problem, the $\frac{\partial \tilde{V}}{\partial n}$ and $\frac{\partial \tilde{B}}{\partial n}$ values obtained from the direct formulation corresponding to a specified slip length and conductivity constant are taken as overspecified boundary conditions in addition to $V=0$ and $B=0$ on the right wall. The DRBEM discretization of the Cauchy MHD duct flow problem leads to an illconditioned system of equations in the form of $A x=b$ due to overspecified conditions on the right wall and underspecified conditions on the left wall. The iterative algorithm developed by Kozlov et al. [15] is used to regularize the ill-posed Cauchy problem including the following steps

Step 1. Solve the matrix-vector equations (7)-(8) in one stroke as a direct problem with the specified $\alpha$ and $c$ values given as in Figure 1 to get the unknown values for $V, B$ on $x=-1$ and their normal derivatives on $x=\mp 1$. Now, the Cauchy MHD problem is constructed with the assumption that the underspecified conditions on $x=-1$ 
(neither $V, B$ nor $\frac{\partial V}{\partial n}, \frac{\partial B}{\partial n}$ are known) and overspecified conditions on $x=1$ ( $V, B$ and their normal derivatives are known).

Step 2. Solve the coupled discretized equation (7)-(8) as a direct problem using the Dirichlet type boundary conditions for $V$ and $B$ on $x=\mp 1$ part of the boundary. The solution gives normal derivatives of $V$ and $B$ everywhere on the boundary.

Step 3. Use $\frac{\partial V}{\partial n}$ and $\frac{\partial B}{\partial n}$ on $x=-1$ and $x=1$ obtained from Step 2 for solving the direct problem to find the new $V, B$ values.

Step 4. Update the values of $V$ and $B$ with the values obtained from step 3 on $x=-1$.

Step 5. Repeat steps 2-4 until the velocity and the induced magentic field values converge to the solution of the direct problem corresponding to the specified slip length $\alpha$ and the conductivity constant $c$.

\section{Numerical results}

In the application of the DRBEM, $N=$ $80,100,160$ constant boundary elements and $L=$ $400,625,1600$ interior nodes are taken for $M=$ $5,10,50$, respectively. In the well-posed iterations, the convergence criteria is taken as $10^{-4}$ as the maximum absolute error in the values of $V$ and $B$, seperately, between the consecutive iterations.

Although there are discontinuities at the corners of the rectangular duct due to the different boundary conditions for $V$ and $B$ on each part of the duct, since the constant boundary elements are used in the numerical solution through the DRBEM, the nodes are not lying on the corners. Hence there is no discontinuity at the corner points numerically (nodes are located at the centers of the constant boundary elements).

Table 1. Velocity and induced magnetic field values from the inverse solution at the point $(-1,0), \quad M=$ $10, \beta=\frac{\pi}{2}, \alpha=0.2, c=0.3$.

\begin{tabular}{|l|c|c|}
\hline $\boldsymbol{N}$ & $\boldsymbol{V}$ & $\boldsymbol{B}$ \\
\hline 44 & 0.0630 & 0.0618 \\
\hline 60 & 0.0623 & 0.0616 \\
\hline 68 & 0.0621 & 0.0616 \\
\hline 76 & 0.0620 & 0.0616 \\
\hline 84 & 0.0619 & 0.0616 \\
\hline 92 & 0.0619 & 0.0616 \\
\hline 100 & 0.0618 & 0.0616 \\
\hline 108 & 0.0618 & 0.0616 \\
\hline 116 & 0.0618 & 0.0616 \\
\hline
\end{tabular}

The mesh independence is shown in Table 1 for Hartmann number $M=10$. The velocity and induced magnetic field values at the point $x=$ $-1, y=0$ which is on the slipping and variably conducting part of the duct are given for several numbers of constant boundary elements. It is seen that when $N$ is taken as larger than $N=100$, the values of $V$ and $B$ are the same up to the order $10^{-4}$. Hence, $N=100$ can be taken as the optimal number of boundary elements for that Hartmann number.

The integrals in the off-diagonal entries of the matrices $\mathrm{H}$ and $\mathrm{G}$ in (9) are calculated by using Gauss-Legendre integration with 16 points. The diagonal entries $G_{i i}$ are evaluated theoretically taking care of the singularities and given in equation (9). $H_{i i}$ are computed implicitly with the assumption of a constant potential over the whole boundary giving zero flux and $H I=0$.

The direct problem is solved when the left wall is taken as slipping and variably conducting by taking the slip length $\alpha=0.2$ and the conductivity constant $c=0.3$. In addition, the external magnetic field is applied with an angle $\beta=0, \frac{\pi}{4}, \frac{\pi}{3}, \frac{\pi}{2}$ formed with the $y$-axis.

The direct solutions for $V$ and $B$ are obtained by using the radial basis functions as $f=1+r$, $f=1+r+r^{2}, f=r^{2} \ln r$ for the angle $\beta=\frac{\pi}{2}$ and the Hartmann number $M=10$. The Figure 2 indicates that there is no significant difference between the solutions, hence because of the simplicity and computational effort, the rest of the calculations are carried by using $f=1+r$.

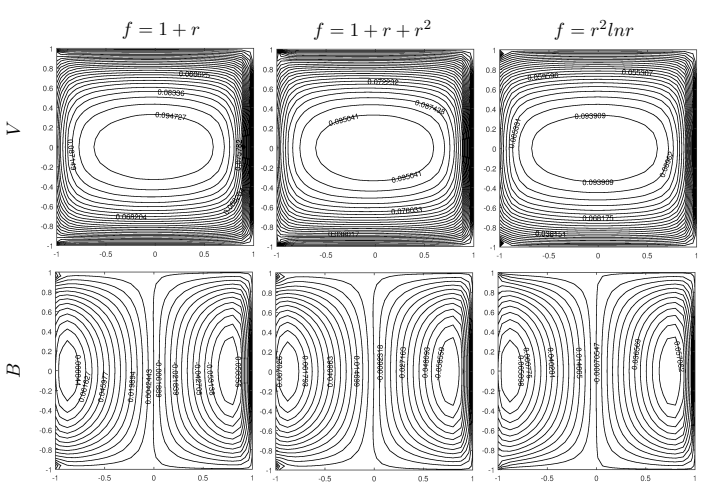

Figure 2. Velocity and induced magnetic field from direct solution, $\beta=\frac{\pi}{2}, \alpha=0.2, c=0.3, M=10$. 


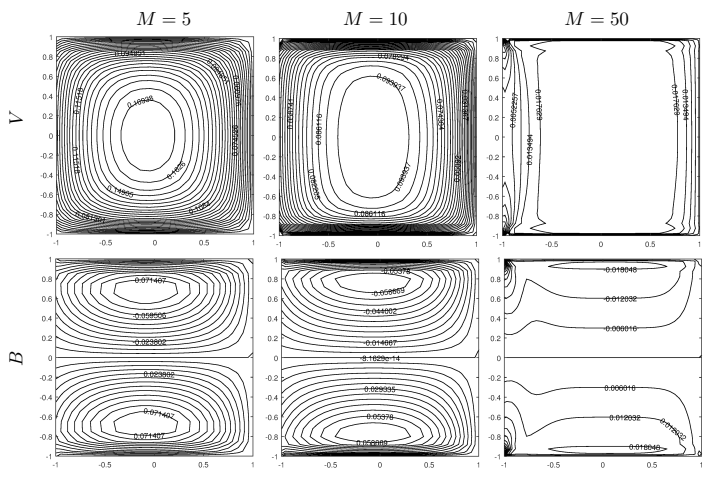

Figure 3. Velocity and induced magnetic field from direct problem, $\beta=0, \alpha=0.2, c=0.3$.

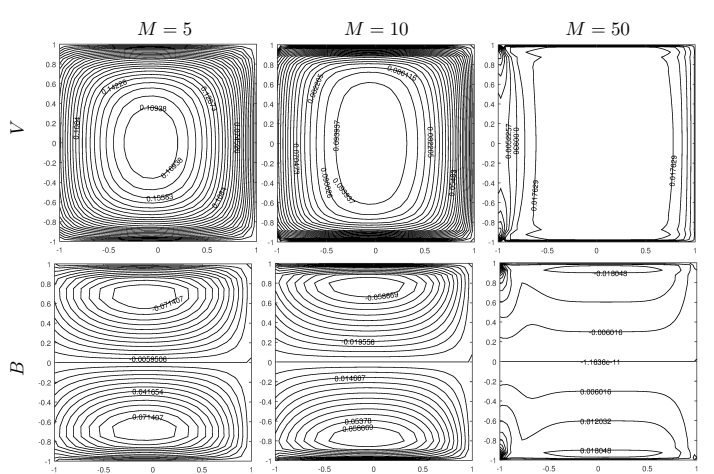

Figure 4. Velocity and induced magnetic field from inverse solution, $\beta=0$.

The direct solutions of MHD flow equations (1) for $V$ and $B$ with the boundary conditions (2) and the slip length $\alpha=0.2$, conductivity constant $c=0.3$ on the left wall are presented for Hartmann number values $H a=5,10,50$ for the angle $\beta=0$ in Figure 3. It is noted that when $\beta=0$, the external magnetic field $B_{0}$ applies in the $y$-direction. It is observed that as $H a$ number increases, Hartmann layers near the top and bottom walls are developed for both $V$ and $B$ which is a well-known behaviour of the MHD duct flow. Also, on the left wall the slip effect is observed which is weakened when $M$ increases. In addition, as $M$ increases, the core region of the fluid enlarges and flow becomes flattened. The induced current seperates into two bunches. Due to the variable conductivity, current lines cross the left walls.

Figure 4 shows the velocity behaviours for the Cauchy MHD flow problem resulted from the well-posed iterations for $M=5,10,50$ and $\beta=0$. It can be seen that the velocity contours obtained from the inverse formulation are in a very well agreement with the ones in the direct problem obtained for $\alpha=0.2$ and $c=0.3$.

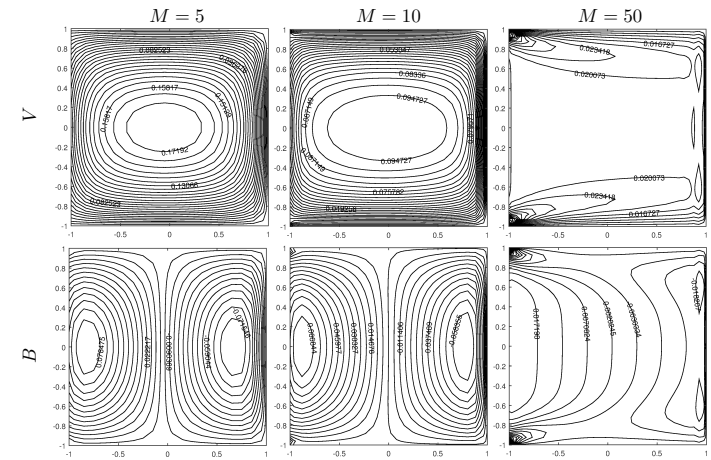

Figure 5. Velocity and induced magnetic field from direct problem, $\beta=\frac{\pi}{2}, \alpha=0.2, c=0.3$.
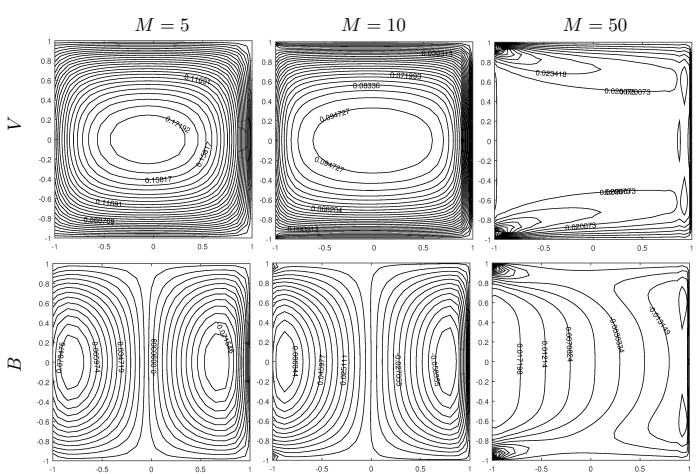

Figure 6. Velocity and induced magnetic field from inverse solution, $\beta=\frac{\pi}{2}$.

In Figures 5,6, the angle $\beta$ is taken as $\beta=\frac{\pi}{2}$, i.e, the external magnetic field applies in the $x$ direction. It is clearly seen that the slip and variable conductivity effect weakens the Hartmann layer near the left wall. A further increase in Hartmann number separates the flow through the top and bottom walls and diminishes the slip phenomenon on the left wall. In addition, the solutions of the direct and inverse problems are in a very well agreement in the sense of fluid movement and the induced current behavior.

Due to the variable conductivity, current lines cross the left walls. An increase in $M$ results in squeezing the right bunch of the induced current through the right wall. 


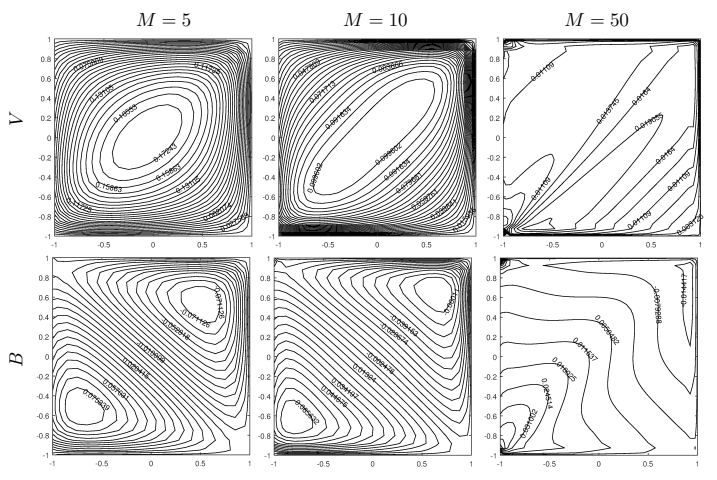

Figure 7. Velocity and induced magnetic field from direct problem, $\beta=\frac{\pi}{4}, \alpha=0.2, c=0.3$.

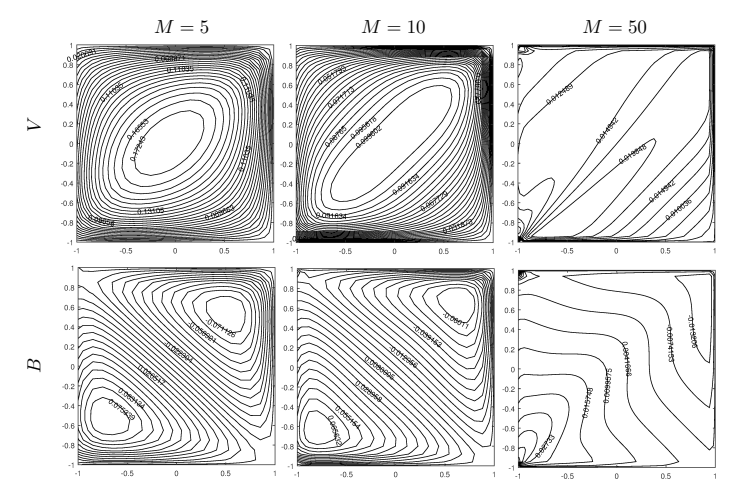

Figure 8. Velocity and induced magnetic field from inverse solution, $\beta=\frac{\pi}{4}$.

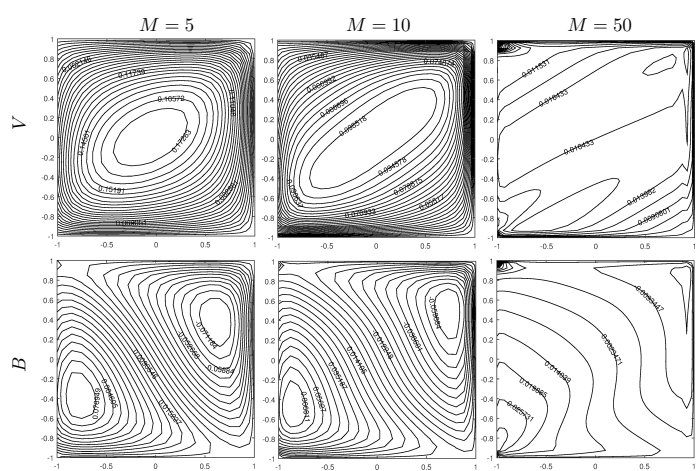

Figure 9. Velocity and induced magnetic field from direct problem, $\beta=\frac{\pi}{3}, \alpha=0.2, c=0.3$.

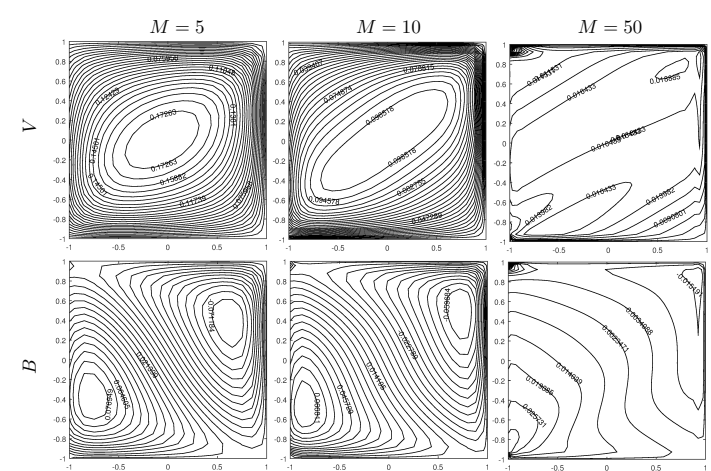

Figure 10. Velocity and induced magnetic field from inverse solution, $\beta=\frac{\pi}{3}$.

When the angle $\beta=\frac{\pi}{4}, \frac{\pi}{3}$ are taken, the direct and inverse solutions for the velocity $V$ and the induced magnetic field $B$ are given in the Figures $6-9$. It is depicted that the core region of the fluid rotates in accordance with the angle $\beta$ with the $y$-axis showing the slip near the left wall. The boundary layers are directed near the corners of the duct in the external applied magnetic field direction showing the well-known behavior of the MHD duct flow. It is observed that the Cauchy MHD flow problem solutions through the wellposed iterations show the very well agreement with the ones in the direct problem solutions for each angle considered.

\subsection{Reconstruction of the slip length and the conductivity constant}

In this study, the main purpose of the Cauchy formulation of MHD duct flow problem is to regain the slip length $\alpha$ and the conductivity constant $c$ on both the slipping and variably conducting part of the boundary. Since the slip condition and variable conductivity condition contain both the velocity, the induced magnetic field and their normal derivative values on the left part of the boundary, one need both of these computed values to extract $\alpha$ from the equation $V+\alpha \frac{\partial V}{\partial n}=0$ and also $c$ from $B+c \frac{\partial B}{\partial n}=0$. The boundary element method is the unique numerical procedure which provides the normal derivative if the solution itself is known or vice versa. Thus, we made use of the knowledge of direct solutions to obtain normal derivative values of the velocity and the induced magnetic field through the Cauchy MHD flow problem solution.

Resized slip length and the conductivity constant on the left wall from the inverse formulations are computed through the relation 
$\alpha=-V / \frac{\partial V}{\partial n}$ and $c=-B / \frac{\partial B}{\partial n}$ where $V, B$ and their normal derivatives are obtained by DRBEM.

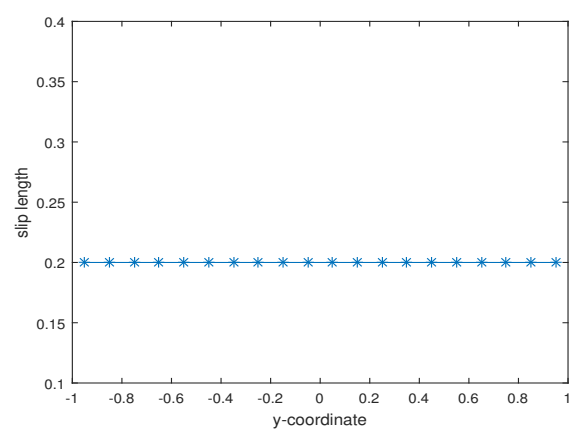

(a) slip length, $\alpha$.

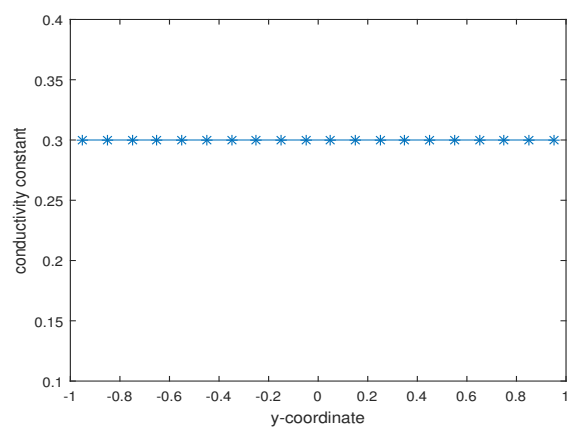

(b) conductivity constant, $c$.

Figure 11. $\beta=\frac{\pi}{4}, x=-1$.

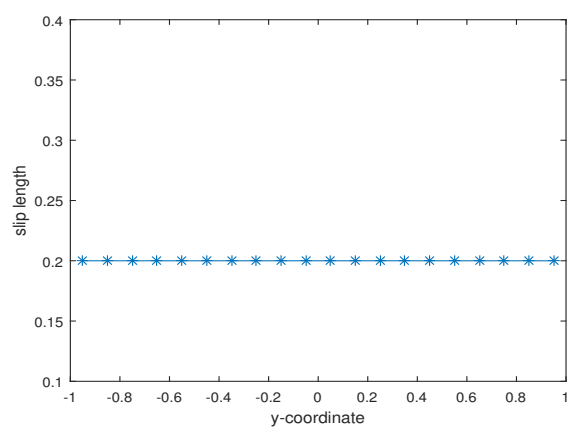

(a) slip length, $\alpha$.

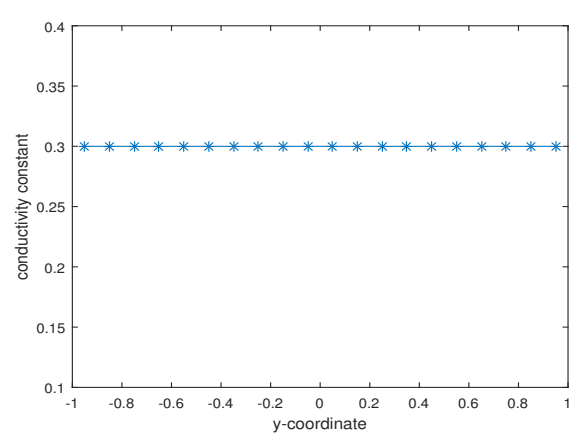

(b) conductivity constant, $c$.

Figure 12. $\beta=\frac{\pi}{3}, x=-1$.

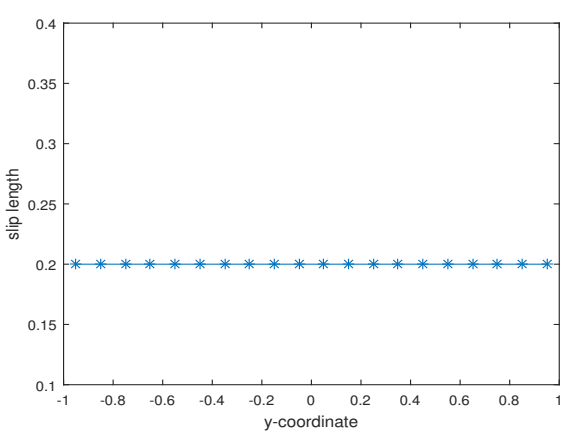

(a) slip length, $\alpha$.

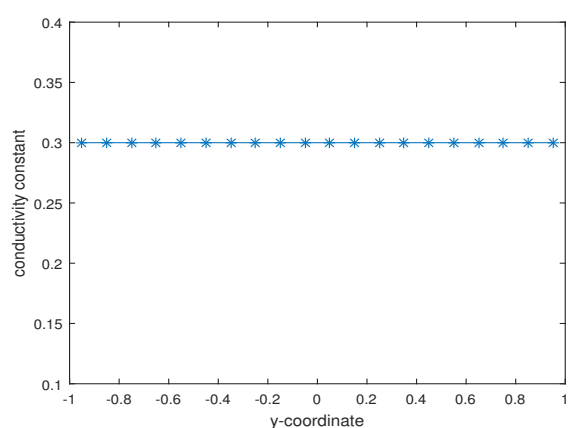

(b) conductivity constant, $c$.

Figure 13. $\beta=\frac{\pi}{2}, x=-1$.

Figures 10-13 show that the slip length $\alpha=0.2$ and the conductivity constant $c=0.3$ are very well approached from the Cauchy MHD flow problem for all of the points on the slipping and variably conducting left wall for $\beta=0, \frac{\pi}{4}, \frac{\pi}{3}, \frac{\pi}{2}$, and $M=5,10,50$ when the well-posed iterations technique is used.

\section{Conclusion}

The direct and Cauchy formulations are constructed for the MHD rectangular duct flow problems in terms of the slip velocity and induced magnetic field on the left wall, and they are solved by using the DRBEM. The Cauchy problems are considered with the normal derivatives of the velocity and induced magnetic field on the right wall obtained from the direct solution to determine the underspecified velocity informations on the left part of the boundary. The well-posed iterations are used to regularize the Cauchy MHD flow problem and the inverse solutions for the velocity and induced magnetic field on the slipping and variably conducting duct wall are obtained for several orientations of the external magnetic field. The Cauchy problems resized the slip velocities and the varying induced magnetic fields using the solution of corresponding direct problem with a preassigned slip length and conductivity constant for $M=5,10,50$. When reconstructing slip 
length and conductivity constant from the wellposed iterations, it is clearly seen that it gives the same estimated slip length and conductivity constant value used in the direct problem. Providing both the velocity, the induced magnetic field and their normal derivative values on the underspecified wall, extracting the slip length $\alpha$ and conductivity constant $c$ between them and discretizing only the boundary of the problem region, the DRBEM is the most appropriate numerical technique for the solution of Cauchy MHD duct flow problems.

\section{References}

[1] Dragoş, L. (1975). Magnetofluid Dynamics. Abacus Press, England.

[2] Shercliff, J.A. (1953). Steady motion of conducting fluids in pipes under transverse fields. Proc. Cambridge Philos. Soc, 49, 136-144.

[3] Ligere, E., Dzenite, I., Matvejevs, A. (2017). Analytical solution of the problem on MHD flow in duct with perfectly conducting Hartmann walls and slip condition on conducting side walls. Proc. of the Engineering for Rural Development. Jelgava.

[4] Ligere, E., Dzenite, I., Matvejevs, A. (2016). MHD Flow in the duct with perfectly conducting Hartmann walls and slip condition on side walls. Proc. of the 10th Pamir International Conference- Fundamental and Applied MHD. Cagliari-Italy.

[5] Aydın, S.H., Tezer-Sezgi, M. (2014). DRBEM solution of MHD pipe flow in a conducting Medium. Journal of Computational and Appllied Mathematics, 259, 720-729.

[6] Bozkaya, C., Tezer-Sezgin, M. (2007). Fundamental solution for coupled magnetohydrodynamic flow equations. Journal of Computational and Applied Mathematics, 203, 125144.

[7] Nesliturk, A.I., Tezer-Sezgin, M. (2006). Finite element method solution of electrically driven magnetohydrodynamic flow. Journal of Computational and Applied Mathematics, 192, 339-352.

[8] Tezer-Sezgin, M., Aydin, S.H. (2006). Solution of MHD flow problems using the boundary element method. Engineering Analysis with Boundary Elements, 30(5), 411-418.

[9] Marin, L., Elliott, L., Heggs, P.J., Ingham, D.B., Lesnic, D., Wen, X. (2006). Dual reciprocity boundary element method solution of the Cauchy problem for Helmholtz-type equations with variable coefficients. Journal of Sound and Vibration, 297, 89-105.

[10] Partridge, P.W., Brebbia, C.A., Wrobel, L.C. (1992). The Dual Reciprocity Boundary Element Method. Computational Mechanics Publications, Southampton, Boston.

[11] Hosseinzadeh, H., Dehghan, M., Mirzaei, D., Aydin, S.H. (2013). The boundary elements method for magneto-hydrodynamic (MHD) channel flows at high Hartmann numbers. Applied Mathematical Modelling, 37(4), 23372351.

[12] Pekmen,B., Tezer-Sezgin, M. (2013). DRBEM solution of incompressible MHD flow with magnetic potential. Computer Modeling in Engineering and Sciences, 96(4), 275-292.

[13] Smolentsev, S. (2009). MHD duct flows under hydrodynamic slip condition. Theoretical and Computational Fluid Dynamics, 23, 557-570.

[14] Aydın, C., Tezer-Sezgin, M. (2018). DRBEM solution of the Cauchy MHD duct flow with a slipping perturbed boundary. Engineering Analysis with Boundary Elements, 93, 94-104.

[15] Kozlov, V.A., Maz'ya, V.G., Fomin, A.F. (1991). An iterative method for solving the Cauchy problem for elliptic equations. Computational Mathematics and Mathematical Physics, 31, 45-52.

Cemre Aydin She received her B.Sc.degree in mathematics from the Middle East Technical University, Turkey in 2014. She is currently a Ph.D student in the Department of Mathematics, Middle East Technical University. Her research is in applied mathematics and numerical analysis focusing especially on the numerical solutions of the Cauchy magnetohydrodynamic flow problems.

Munevver Tezer-Sezgin She holds B.Sc. and M.Sc. degrees in mathematics from the Middle East Technical University, Turkey, M.Sc. and Ph.D degrees in Applied Mathematics from the University of Saskatchewan and the University of Calgary, Canada. Currently, she is a professor in the Department of Mathematics and an affiliated member of the Institute of Applied Mathematics, Middle East Technical University. She has held visiting positions at the University of Victoria, Canada, and Technical Univerity of Darmstadt. Her research is mainly in numerical analysis applied to fluid dynamics and magnetohydrodynamics. She has published over 130 research articles in specialized international journals and the international conference proceedings. She is the recepient of the "Mustafa Parlar Foundation 1990 Research Prize" and "Mustafa Parlar Foundation 2014 Science Prize" in applied mathematics and engineering. 
An International Journal of Optimization and Control: Theories \& Applications (http://ijocta.balikesir.edu.tr)

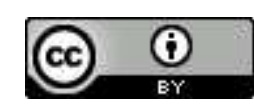

This work is licensed under a Creative Commons Attribution 4.0 International License. The authors retain ownership of the copyright for their article, but they allow anyone to download, reuse, reprint, modify, distribute, and/or copy articles in IJOCTA, so long as the original authors and source are credited. To see the complete license contents, please visit http://creativecommons.org/licenses/by/4.0/. 